\title{
Free Energy Evolution and Photon Radiation from QGP
}

\author{
Yogesh Kumar and S. Somorendro Singh \\ Department of Physics and Astro-Physics, University of Delhi, Delhi-110007, India \\ Correspondence should be addressed to Yogesh Kumar; yogesh.du81@gmail.com
}

Received 12 July 2013; Accepted 29 August 2013

Academic Editors: M. Masip and Z. H. Xiong

Copyright (C) 2013 Y. Kumar and S. S. Singh. This is an open access article distributed under the Creative Commons Attribution License, which permits unrestricted use, distribution, and reproduction in any medium, provided the original work is properly cited.

\begin{abstract}
We investigate a simple statistical model of quark-gluon plasma (QGP) formation. In the model, we use a phenomenological parameter which enhances the growth of quark droplet formation and also stabilizes the formation of the QGP droplet. Then, we study direct photon radiation through annihilation and Compton processes from these stabilized QGP incorporating the parametrized momentum factor in the quark mass. The production rate of thermal photon is found to be dominated in the low transverse momentum and increases a little in comparison to the recent development of direct photon radiation of other theoretical and experimental works.
\end{abstract}

\section{Introduction}

The main objective idea of ultrarelativistic heavy-ion collisions is to create and study strongly interacting matter which is obtained from transformation of a confined matter of hadronic state to a deconfined state of free quarks and gluons $[1,2]$. The interacting matter is found to exist in the form of a superfluid state called quark-gluon plasma (QGP) and its existence is reported at Relativistic Heavy IonCollision (RHIC) and at Large Hadron Collider (LHC) [36]. Moreover, the report from lattice QCD calculation proves the existence of such matter (QGP) at very high temperature [7]. In fact, experiments at SPS/CERN, RHIC/BNL, and LHC/CERN may create such situation at high temperature for the study of QGP [8]. It is also observed experimentally that the formation of QGP is done due to central collision of two massive nuclei. So, the experiments at heavy-ion reaction are really trying to create the same condition that existed at the time of creation of the early universe, that is, the condition just after the big-bang. To prove the existence of the matter, there are various possible probes of QGP formation. Among these probes, the experimental measurement of electromagnetic observables in heavy-ion collision gives one of the most promising and exciting signals in heavy-ion reactions due to their noninteraction after their productions. These are considered to be highly energetic photons and leptons [917] and have been recognized as direct probes of collisions.
The electromagnetic signals have large mean-free path at their emission. Due to the large mean-free path, they interact as electromagnetic reactions and leave the fireball undisturbed, and they carry all the information about existence of the plasma. So, they give the fundamental information for the formation of QGP.

So far, many investigations have been devoted to the research of hard photon production from quark-gluon plasma at finite temperature [18-21]. The authors first consider the ideal fluid dynamics to study the subsequent evolution of kinematically equilibrated QGP phase at very high energy and temperature. Moreover experimental observations [22-26] from $\mathrm{Pb}-\mathrm{Pb}$ collision at $\sqrt{s}=158 \mathrm{AGeV}$ by CERN WA80 and WA98 collaboration at SPS indicate the first favorable interest in these electromagnetic probes. They further expect data from RHIC and LHC [27]. These observations show the production of excess direct photon from the results of hadronic decays in the intermediate transverse momentum region of the most central collisions. This supports the confirmation of direct photon as a feasible signal for the formation and evolution of QGP.

Furthermore, the current experimental programs at ultrarelativistic heavy-ion collisions at RHIC/BNL and LHC/CERN have indicated the main goal of its experimental pursuit in the research of QGP with the higher beam energies. The active program will provide deeper understanding in the analysis of theoretical modelling. In addition to these 
ideas, the transverse momentum distribution function of direct photon is significant in the photon induced reaction at the same energies $\sqrt{s}$ [28-30] for the momentum greater than $1.5 \mathrm{GeV} / \mathrm{c}$ in the central collision, which implies the importance of direct photon production dependent on the space time evolution scenarios of the finite QGP formation. On these information, we focus the photon radiation directly from the thermalized quark-gluon plasma at $T=0.25 \mathrm{GeV}$ which expands and cools, comes back to hadronic matter with the production of latent heat, that again heats and cools, and eventually freezes out at last into hadrons such as pions at around temperature $T=0.15 \mathrm{GeV}[31]$.

In the present work, we investigate a simple model to create the formation of these strongly interacting matter of quark-gluon plasma with the parametrized momentum factor in the quark mass. The thermal dependent quark mass removes the infrared (IR) divergence in production rate of photon that occurred in the dominant reaction of annihilation and Compton processes. There are infrared divergences in the photon production rate with the massless quark. So, quark mass is used in removing the IR divergence and it is initially handled by the technique of [32-34]. So, we consider the thermal finite quark mass as [35]

$$
m_{q}^{2}=\frac{1}{3} \frac{8 \pi}{\left(33-2 n_{f}\right)} \frac{1}{\ln \left(1+\left(q^{2} / \Lambda^{2}\right)\right)} T^{2},
$$

where $q=\left(\left(\gamma N^{(1 / 3)} T^{2} \Lambda^{2}\right) / 2\right)^{1 / 4}$ is known as low momentum cut-off with $N=16 \pi /\left(33-2 n_{f}\right)$ and parametrization factor $\gamma=\sqrt{2} \sqrt{1 / \gamma_{q}^{2}+1 / \gamma_{g}^{2}}$ with $\gamma_{g}=a \gamma_{q} . a$ is either 6 or 8 with $\gamma_{q}=1 / 6[36,37]$. These factors are suitably chosen in an ad-hoc fashion to fit the free energy evolution of the QGP.

Now, we study photon radiation at the different temperatures for the two different quark flavors $n_{f}=2$ and 3 . We also calculate the photon production and total spectra at the hot phase of QGP evolution at the temperatures $T=0.25$ and $0.30 \mathrm{GeV}$ and compare the results with other theoretical and experimental results.

Thus, we organize the paper as follows. In Section 2, we give a brief idea of free energy evolution of QGP. In Section 3, we present the photon radiation and total spectra from QGP, and in Section 4, we give the results and conclusion.

\section{Free Energy Evolution of QGP}

The free energies of the constituent particles of QGP evolution can be calculated through density of states of the noninteracting fermions and bosons at the finite temperature. The calculated free energies of quarks and gluons are defined as follows [38-41]:

$$
F_{i}=\mp T g_{i} \int d q \rho_{i}(q) \ln \left(1 \pm e^{-\sqrt{m_{i}^{2}+q^{2}} / T}\right),
$$

where $g_{i}$ is degeneracy factor and $\rho_{i}(q)$ is density of states of the constituent particles of the QGP. The density of state is obtained through the mean field potential among quarksantiquarks and quarks-gluons. To find the potential, we take the thermal Hamiltonian process of the thermal dependent quark mass. The Hamiltonian is defined as [42]

$$
H(q, T)=H_{o}(q, T)+\frac{1}{2 q} \gamma_{q, g} g^{2}(q) T^{2},
$$

where unperturbed Hamiltonian is

$$
H_{o}(q, T)=\left[q+\frac{m_{o}^{2}}{2 q}\right]
$$

and the second one is considered to be the effective potential term in which $g^{2}(q)=4 \pi \alpha_{s}$ is a QCD coupling constant with $\alpha_{s}$ defined as

$$
\alpha_{s}=\frac{4}{\left(33-2 n_{f}\right) \ln \left(1+\left(q^{2} / \Lambda^{2}\right)\right)} .
$$

We construct the density of state of quarks and gluons by adapting the Thomas-Fermi model for electrons and Bethe model of nucleons as templates [43-45] through this potential of the Hamiltonian and it is, therefore, defined as

$$
\rho(q)=\frac{v^{2} \gamma^{3} T^{6}}{4 \pi^{2} q^{3}} g^{4}(q)\left[\frac{d g(q)}{d q}-\frac{g^{2}(q)}{2 q}\right] .
$$

In addition to the above free energy, there are energy contributions in the evolution of the QGP fireball from the Weyl surface energy and pions. During the formation of quark and gluon droplets, surface energy due to Weyl surface [46] has considerable amount of energy contribution in the formation of droplets which is taken as

$$
F_{\text {surface }}=\gamma T \int d q \rho_{\text {weyl }}(q) \delta(q-T),
$$

where

$$
\rho_{\text {weyl }}(q)=\left[\frac{4 \pi R^{2}}{(16 \pi)}\right] q^{2} .
$$

In another term, the Weyl energy replaces the role of bag energy of the MIT model. In the above Weyl density, $R$ is the size of the droplets. The contributed pion-free energy is defined as [38-41]

$$
F_{\pi}=\left(\frac{3 T}{2 \pi^{2}}\right) v \int_{0}^{\infty} q^{2} d q \ln \left(1-e^{-\sqrt{m_{\pi}^{2}+q^{2}} / T}\right) .
$$

The process of the transition from QGP to hadronic phase involves pions-free energy for the accurate growth of the QGP fireball. So, the total free energy of the QGP can be computed as

$$
F_{\text {total }}=\sum_{i} F_{i}
$$

where $i$ denotes the contribution of each particle. 


\section{Photon Production from QGP}

The calculation of photon production from QGP is found to be a very interesting theoretical problem. In the creation of early stage of the universe, there was little understood about photon production as the system was just before the process of thermalization. We consider the thermalized state of QGP system after big-bang process and the system take a longer time compared to the time scale associated with the photon production. Moreover, it is stated $[47,48]$ that for the coupling parameter $\alpha_{s} \ll 1$, the result turns out to be slow expansion near the equilibrium temperature, even though it is not justified condition. Resulting by this, there are good number of research works in photon production from the quark-gluon plasma considering the QCD quark antiquark annihilation process and Compton process. Based on these results, we focus the photon production rate and total spectra at thermal equilibrium temperature $T=0.25 \mathrm{GeV}$ and $T=$ $0.30 \mathrm{GeV}$. We calculate subsequently the production rate of photon in the similar line of one loop calculation of Compton process $q(\bar{q}) g \rightarrow q(\bar{q}) \gamma$ plus annihilation $q \bar{q} \rightarrow g \gamma$ and calculate photon production from the quark matter with two loops considerations evaluated by Aurenche et al., and that is known as Bremsstrahlung processes. Finally, we consider the dominant contribution of $q \bar{q}$ annihilation with scattering obtained by Aurench et al., and that is known as AWS.

The formulation of photon production with one loop order of Compton plus annihilation is given by the expression $[14,15,18-21,49]$ :

$$
E \frac{d N}{d^{4} x d^{3} q}=\frac{1}{2 \pi^{2}} \alpha \alpha_{s} \sum_{f} e_{f}^{2} T^{2} e^{-E / T}\left[\ln \left(\frac{4 E T}{q_{c}^{2}}\right)-1.415\right],
$$

where $q_{c}^{2}=2 m_{q}^{2}$. The summation is over the number of quark flavors and $e_{f}$ is their electric charge. Similarly, the production rate of photons due to two loops order of Bremsstrahlung processes is given as [50-53]

$$
E \frac{d N}{d^{4} x d^{3} q}=\frac{8}{\pi^{5}} \alpha \alpha_{s} \sum_{f} e_{f}^{2} \frac{T^{4}}{E^{2}} e^{-E / T}\left(J_{T}-J_{L}\right) I(E, T),
$$

where $I(E, T)=3 \zeta(3)+\pi^{2} E / 6 T+(E / T)^{2} \ln (2)+$ $4 L i_{3}\left(-e^{-|E| / T}\right)+2(E / T) L i_{2}\left(-e^{-|E| / T}\right)-(E / T)^{2} \ln \left(1+e^{-|E| / T}\right)$ and $L i^{\prime} s$ are the polylogarithmic functions given by

$$
L i_{a}(z)=\sum_{n=1}^{\infty} \frac{z^{n}}{n^{a}}, \quad \zeta(3)=1.202 .
$$

In the above expression, $J_{L}=-1.064$ and -1.130 for quark flavors $n_{f}=2$ and 3. Similarly, the values for $J_{T}$ at $n_{f}=2$ and 3 are taken as 1.108 and 1.20 .

Finally, we calculate the most dominant contribution given by $q \bar{q}$ annihilation with scattering process (AWS). The expression for production rate of annihilation with scattering is given as [50-53]

$$
E \frac{d N}{d^{4} x d^{3} q}=\frac{8}{3 \pi^{5}} \alpha \alpha_{s} \sum_{f} e_{f}^{2} E T e^{-(E / T)}\left(J_{T}-J_{L}\right) .
$$

Furthermore, we study the total photon spectrum at temperature $T=0.30 \mathrm{GeV}$ by integrating the total rate over the space-time history of the collision for all the processes after getting the temperature of the evolution from the model. We perform the integration at $T=0.30 \mathrm{GeV}$ as done in other theoretical models. It is expressed as $[54,55]$

$$
\begin{aligned}
\frac{d N}{d^{2} q_{T} d y} & =\int d^{4} x\left(E \frac{d N}{d^{3} q d^{4} x}\right) \\
& =Q \int_{\tau_{0}}^{\tau_{f}} \tau d \tau \int d y\left(E \frac{d N}{d^{3} q d^{4} x}\right),
\end{aligned}
$$

where $\tau$ is the initial and final value of time evolution. We take rapidity $y_{\text {nuc }}= \pm 5.3$ corresponding to RHIC energy. The transverse cross-section of the considered nuclei is taken as $Q \sim 180 \mathrm{fm}^{2} \cdot q_{T}$ is the photon transverse momentum. Then, the quantity on the R. H. S. is defined in the centreof-mass system with the photon energy $E=q_{T} \cosh \left(y^{\prime}-\right.$ $y$ ). Moreover, the longitudinal expansion is assumed to be a dominant factor in the early stage in comparison to the transverse expansion $[56,57]$. The influence of transverse expansion can be considered in later space time evolution around the time $\tau=2.7 \mathrm{fm} / \mathrm{c}$. The production rate will be approximately down in the scale of consistent magnitude with the experimental result and with the inclusion of transverse expansion. So our approximation is well valid during the early stage where our interest lies. Thus, with the values of rapidity and $q_{T}$, we get the total photon spectrum.

\section{Results and Conclusion}

In the results and conclusion we present the numerical results for the evolution of free energy of plasma and photon production rate and also total spectra from the plasma through the different channels at finite temperature with the introduction of parametrization value in the quark mass. The calculation is done for two different quark flavors. The free energy evolution is shown by Figures 1 and 2. In Figure 1, droplet size of QGP formation is small with the quark flavor $n_{f}=2$ but it shows a clear cut information about the stability of the droplet for the various values of temperature at the droplet size of $R=3.5 \mathrm{fm}$. Similarly, we plot free energy with the size of droplet in Figure 2. The droplet size is found to be still large compared to the former one with the proper stability in the droplet formation with the quark flavor $n_{f}=3$ at the same values of temperature. The increased droplet size is found to be $R=7.0 \mathrm{fm}$. The stable-sized droplet is around double times the size of the former one of flavor $n_{f}=2$. It indicates that the use of parametrization in the quark mass in both figures enhances the free energy evolution and creates stability in the droplet size formation of the plasma evolution. The evolution at such stable droplet can produce the photon in the range of free energy $0-6.0 \mathrm{GeV}$. If we increase the temperature $T>250 \mathrm{MeV}$, then the free energy increases with unstable droplet and the photon production will be more with the temperature. But we perform the photon production for stable QGP with suitable choice of parameter 


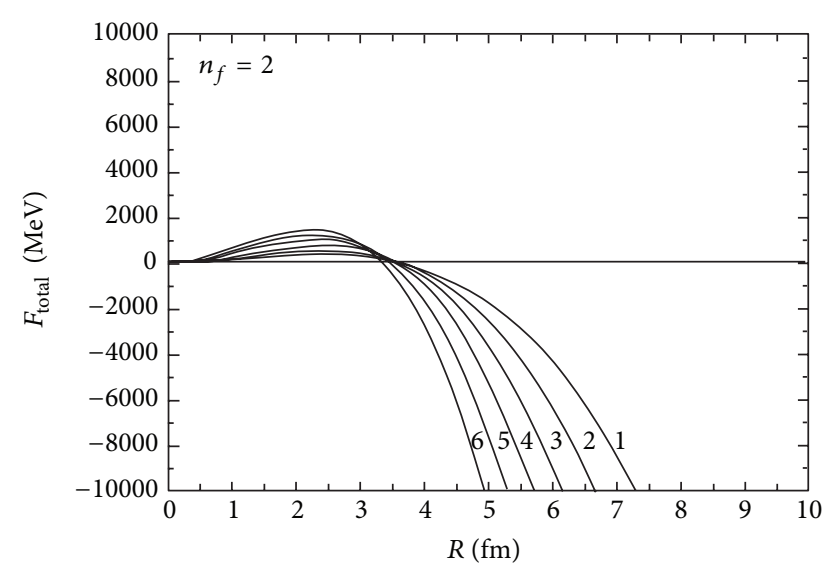
(1) $T=150 \mathrm{MeV}$
(4) $T=210 \mathrm{MeV}$
(2) $T=170 \mathrm{MeV}$
(5) $T=230 \mathrm{MeV}$
(3) $\mathrm{T}=190 \mathrm{MeV}$
(6) $T=250 \mathrm{MeV}$

FIGURE 1: The free energy $F(\mathrm{MeV})$ of QGP evolution versus droplet size $R(\mathrm{fm})$ for thermal temperatures at $n_{f}=2$ with the quark mass.

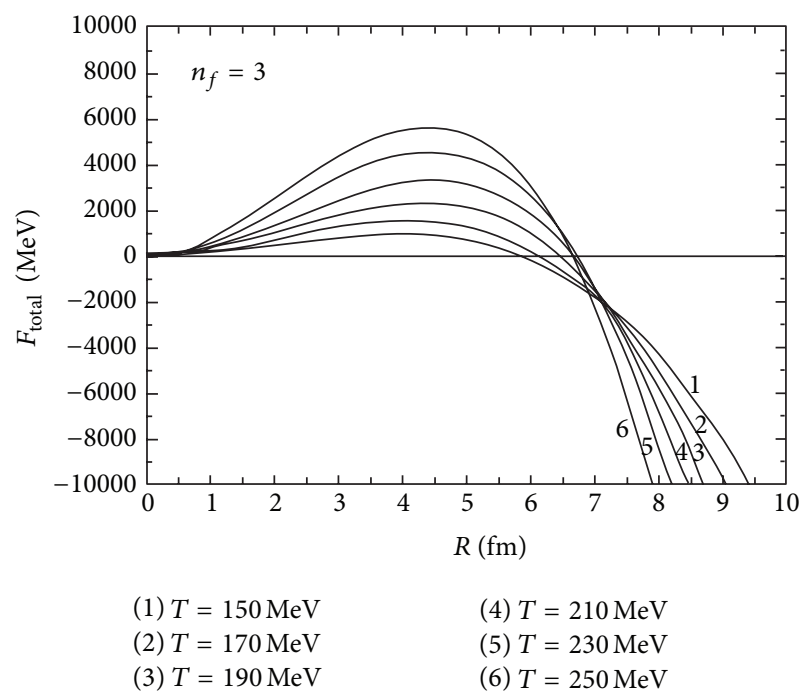

Figure 2: The free energy $F(\mathrm{MeV})$ of QGP evolution versus droplet size $R(\mathrm{fm})$ for thermal temperatures at $n_{f}=3$ with the quark mass.

for annihilation and Compton processes at the range of the free energy forming stable droplet.

In Figures 3 and 4, we show the photon emission rate at the different initial temperatures with the two different quark flavors for the process of annihilation plus Compton processes. The emission rates are found to be increasing with the temperature of the system in both situations of quark flavor. The increase in the emission rate is highly effected by the temperature of the system, and it seems to be large near the creation of quark matter that is found at the very early universe. Besides, there is less suppression in the photon emission with the increase in the photon energy at temperature $T=0.57 \mathrm{GeV}$. This is because of the fact that

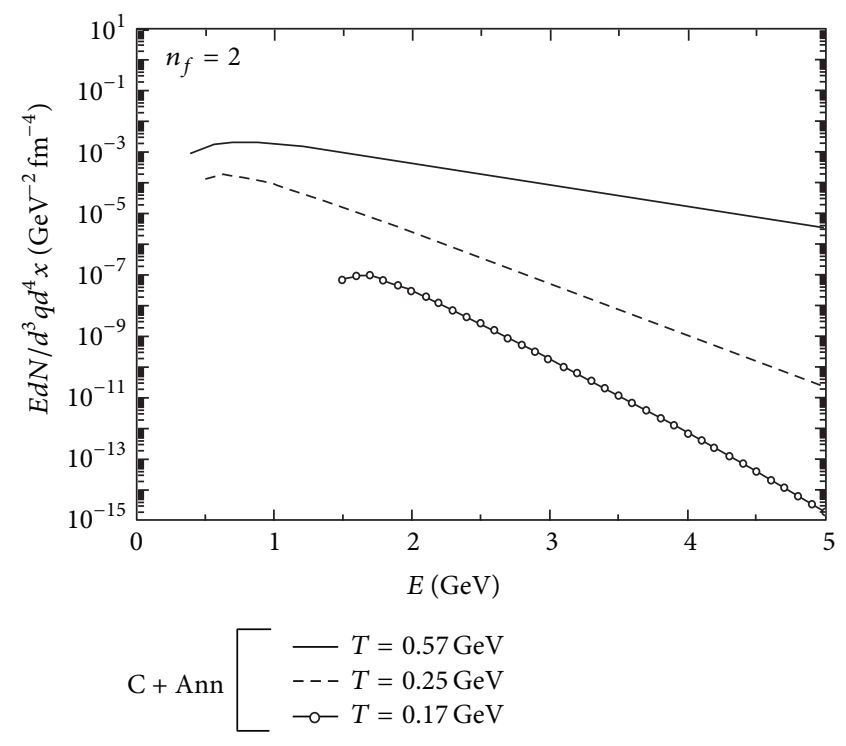

FIgURE 3: The photon emission rate through ann. plus Compton at thermal temperatures $T=0.57,0.25$, and $0.17 \mathrm{GeV}$ with the photon energy for $n_{f}=2$.

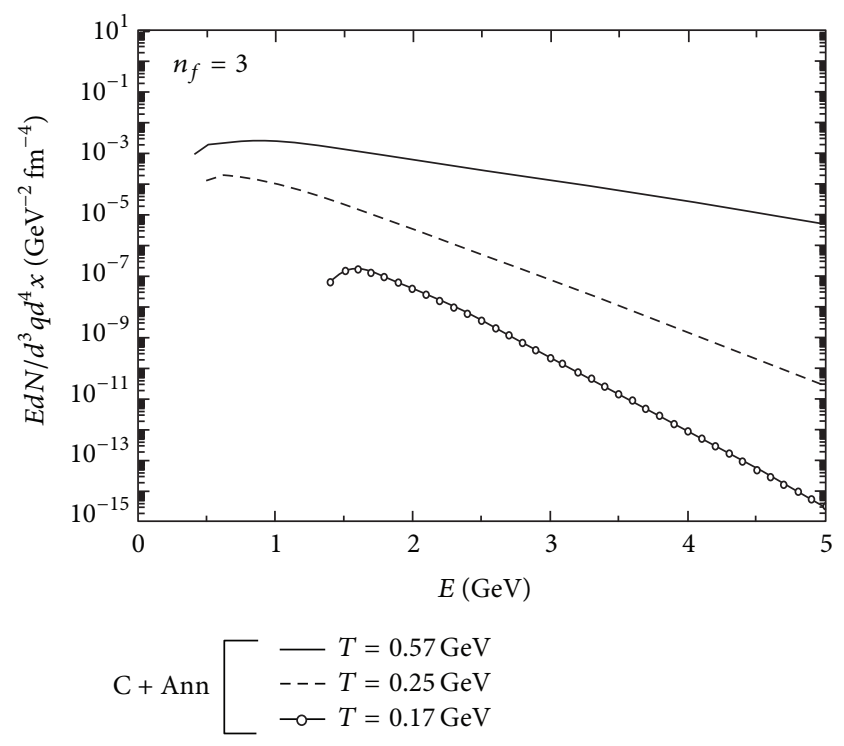

FIGURE 4: The photon emission rate through ann. plus Compton at thermal temperatures $T=0.57,0.25$, and $0.17 \mathrm{GeV}$ with the photon energy for $n_{f}=3$.

the Boltzmann factor $e^{-E / T}$ in the expression (11) does not suppress the yield rate as we increase the energy $E \gg T$. Then, in Figures 5 and 6, we show the compared results of photon emission at temperature $T=0.25 \mathrm{GeV}$ with other theoretical results for the different mechanisms of the photon production. We find that the contribution of Bremsstrahlung is effectively high in the low photon energy $E<1.0 \mathrm{GeV}$ in comparison to the other two channels namely AWS and annihilation plus Compton, and it is dominated by the other two channels in the higher energy for both flavors. Between the two channels, AWS channel has more photon production 

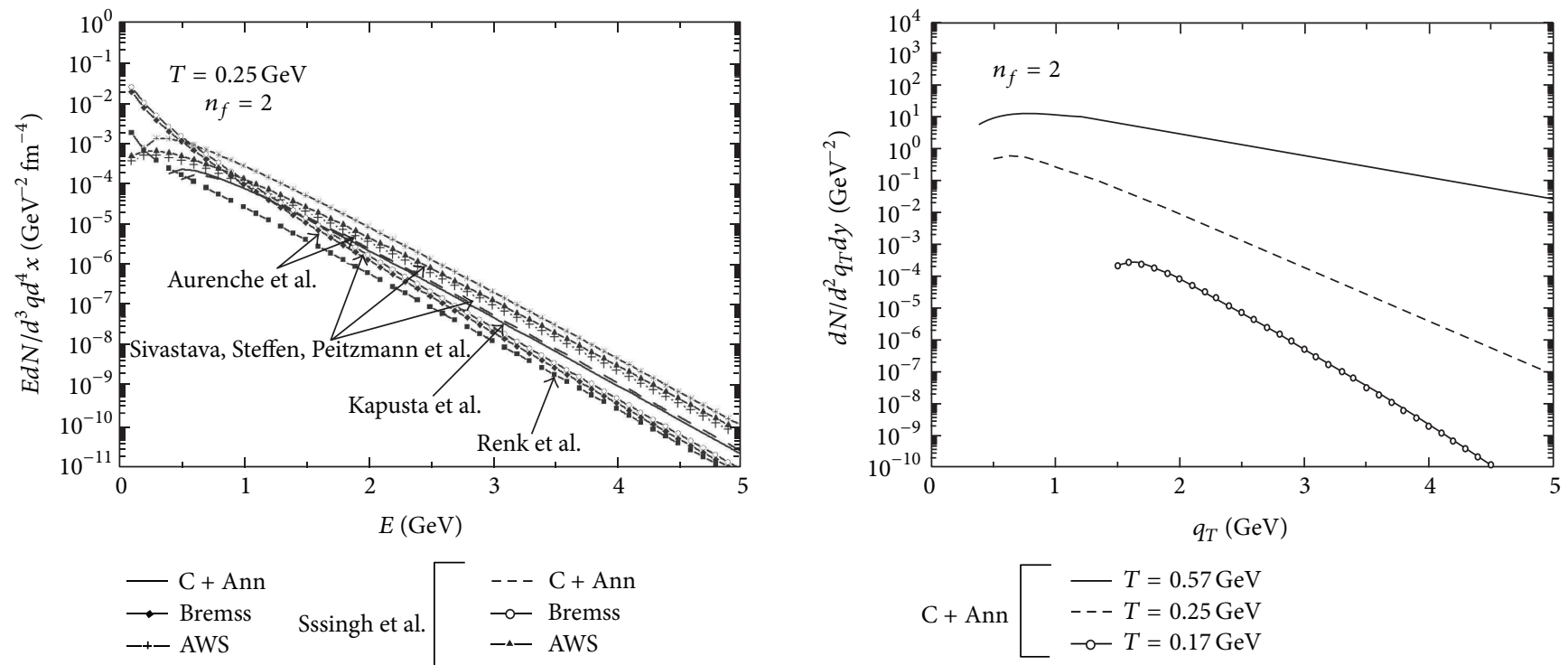

FIgURE 5: The photon emission rate through different channels at thermal temperature $T=0.25 \mathrm{GeV}$ for $n_{f}=2$ and compared with other works.

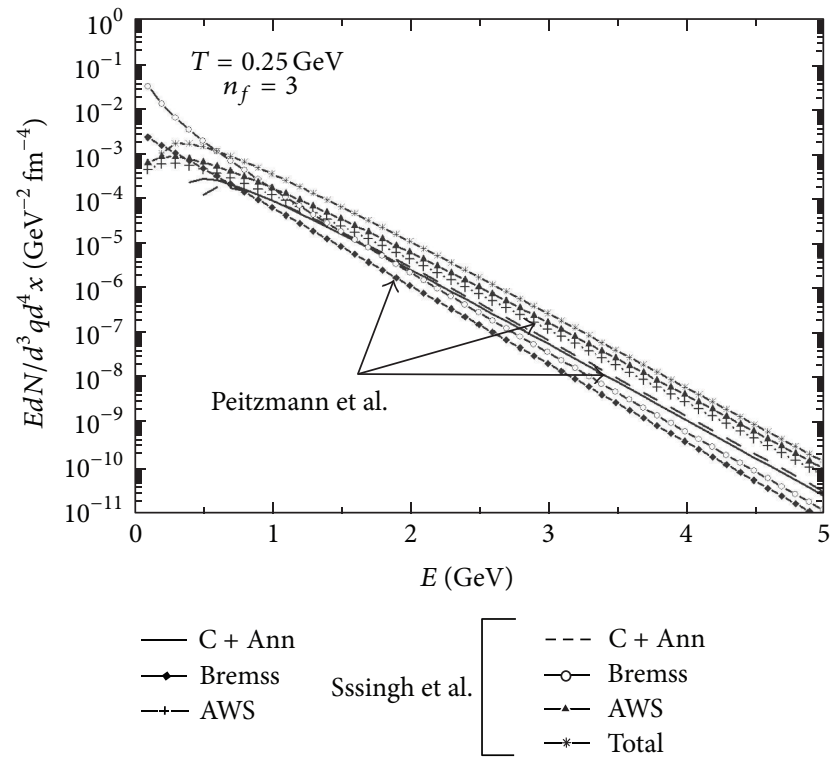

FIgURE 6: The photon emission rate through different channels at thermal temperature $T=0.25 \mathrm{GeV}$ for $n_{f}=3$ and compared with other works.

rate than that of the annihilation plus Compton channel. In all the processes/channels, our model has large advantage in the photon production rate particularly in Bremsstrahlung and AWS, but the production rate has negligible amount of increment in annihilation plus Compton process. A visible increment in AWS and Bremss of flavor $n_{f}=2$ can be seen in Figure 5. Thus, the production rate of our model with the flavor $n_{f}=2$ has improved from other works in these two channels. It means that our result is dominant over the production rate of $[14,15,18,19,50-55]$.

Figure 7: The photon emission rate through ann. and Compton processes at thermal temperatures $T=0.57,0.25$, and $0.17 \mathrm{GeV}$ for $n_{f}=2$.

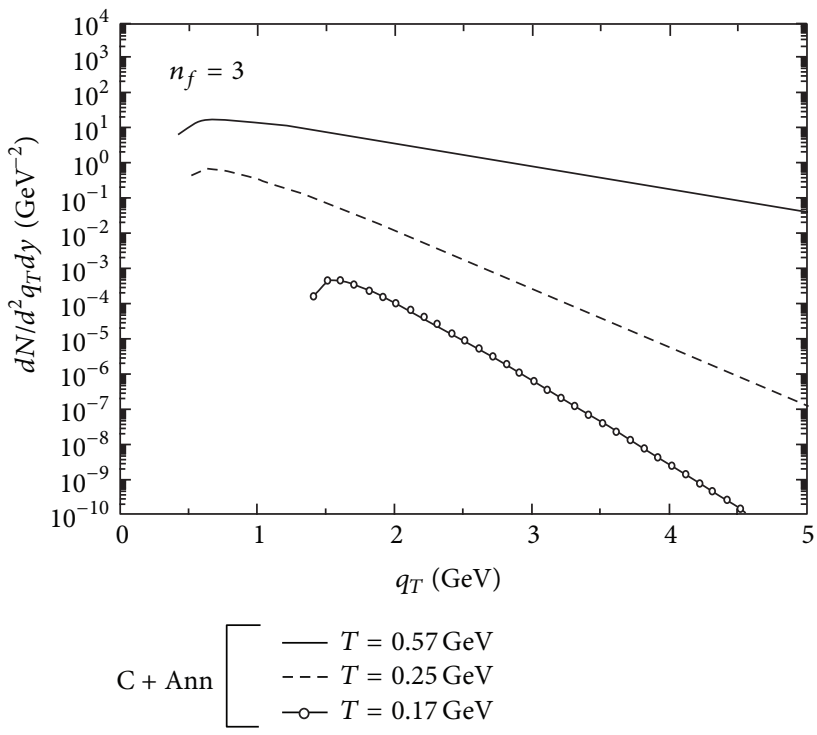

FIgURE 8: The photon emission rate through ann. and Compton processes at thermal temperatures $T=0.57,0.25$, and $0.17 \mathrm{GeV}$ for $n_{f}=3$.

The production rate is further examined for the quark flavor $n_{f}=3$ in Figure 6. In the figure, we compare the outputs between our result and other standard results of $[14,15]$. The result has a visible incremental amount in the production rate in all the three channels. It indicates that the calculation through our model of parametrization value in the quark mass improves in the result of photon productions. The production rate has still enhanced result in comparison to WA98 experiments as well as in many of other theoretical calculations of photon productions and it is shown in Figures 9 and 10. 


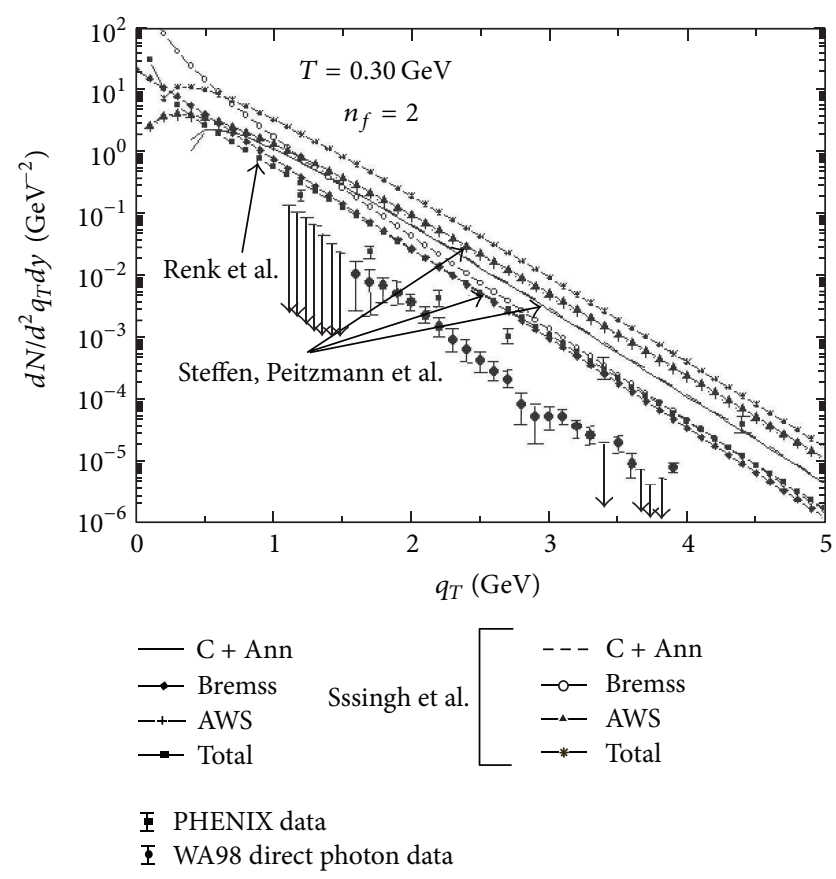

FIGURE 9: The photon emission rate through the different channels at thermal temperature $T=0.30 \mathrm{GeV}$ for $n_{f}=2$ and compared with the other works and experimental data.

Now we study the total photon spectrum over the whole space-time evolution of QGP. These results are also shown in Figures 7-10. In Figures 7 and 8, we first show the total spectra of the photon at different temperatures for $n_{f}=2$ and 3 as a function of photon transverse momentum. In both figures, the production is not much suppressed at the higher energy $E \gg T$ due to the Boltzmann factor in the total yield expression. The figures predict that production rate as function of momentum transverse have much enhancement over the production rate as function of the photon energy.

In comparison of the yield rate of the two different quark flavors, the higher quark flavor has larger yield than the lower value. It is due to more interaction among the large number of constituent quarks.

Lastly, in Figures 9 and 10, we study the total spectra at the temperature $T=0.30 \mathrm{GeV}$ as discussed in other works for the different processes of photon spectra for these two different flavors. In these processes, we compare the results with the earlier outputs of other works $[14,15,50-$ 55] and also compare results with WA98 direct photon data and photon spectra at RHIC energies measured by PHENIX collaboration [22-26, 58-63]. In the comparison, we find that the production spectra of our model in the quark flavor $n_{f}=2$ has negligibly visible improvement over the other recent theoretical result of photon spectra in all the corresponding channels like annihilation plus Compton and AWS, but a slight visible improvement is found in the case of Bremsstrahlung. A similar situation is obtained in the case of quark flavor $n_{f}=3$. This means that the total photon spectra with the model of parametrization has same negligible enhancement over other theoretical calculation and good enhancement over experimental data of photon spectra in
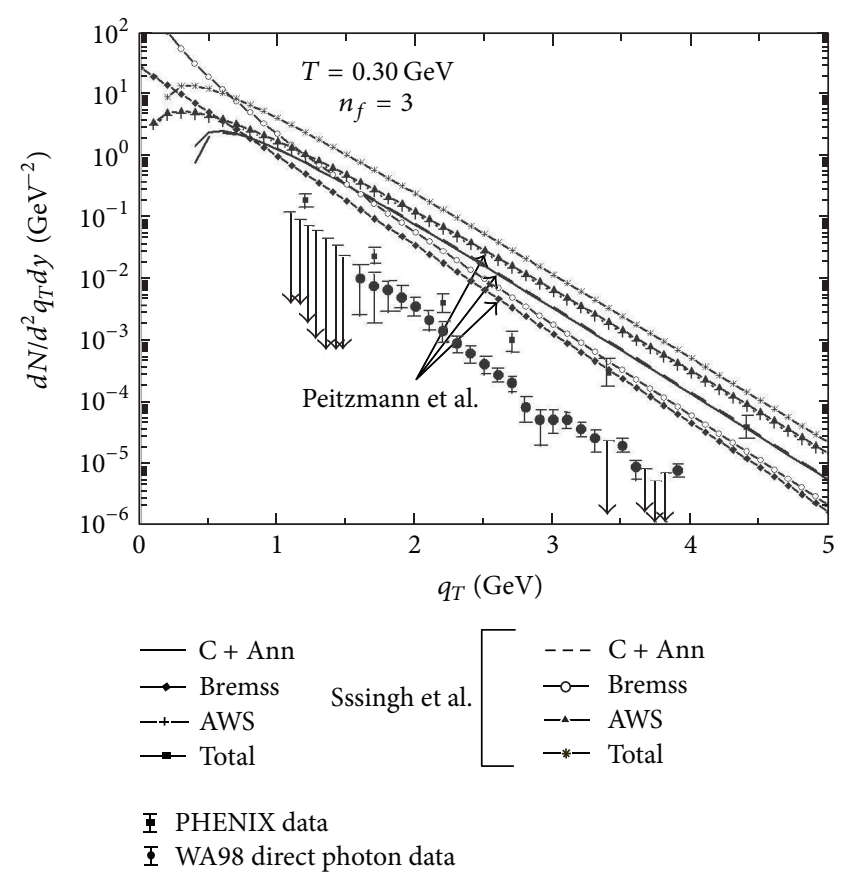

FIGURE 10: The photon emission rate through the different channels at thermal temperature $T=0.30 \mathrm{GeV}$ for $n_{f}=3$ and compared with other works and experimental data.

all the possible processes except the Bremsstrahlung. Above all, it indicates that our result gives significant contributions over other theoretical works and also results from WA98 and PHENIX data collaboration [22-26, 58-63].

Finally, we conclude that the evolution of the fireball through the parametrization factor enhances the stability of the droplet formation. The overall calculation of photon production and total spectra as a function of photon energy and transverse momentum incorporating the factor gives the improved results in the photon yield products from the earlier results. Thus, the consideration of parametrization factor in the quark mass has an important role in the evolution as well as in the photon measurements of the high energy heavy-ion collisions. So, this simple model gives improved results over the other theoretical models and experimental results.

\section{Conflict of Interests}

The authors declare that they have no conflict of interests.

\section{Acknowledgment}

The authors are very thankful to R. Ramanathan for the constructive suggestions and discussions in preparing the paper.

\section{References}

[1] J. W. Harris and B. M. Müller, "The search for the quark gluon plasma," Annual Review of Nuclear and Particle Science, vol. 46, pp. 71-107, 1996. 
[2] K. Geiger and B. M. Müller, "Dynamics of parton cascades in highly relativistic nuclear collisions," Nuclear Physics B, vol. 369, no. 3, pp. 600-654, 1992.

[3] I. Arsene, I. G. Bearden, D. Beavis et al., "Quark-gluon plasma and color glass condensate at RHIC? The perspective from the BRAHMS experiment," Nuclear Physics A, vol. 757, no. 1-2, pp. 1-27, 2005.

[4] B. B. Back, M. D. Baker, M. Ballintijn et al., "The PHOBOS perspective on discoveries at RHIC," Nuclear Physics A, vol. 757, no. 1-2, pp. 28-101, 2005.

[5] J. Adams, M. M. Aggarwal, Z. Ahammed et al., "Experimental and theoretical challenges in the search for the quark-gluon plasma: the STAR Collaboration's critical assessment of the evidence from RHIC collisions," Nuclear Physics A, vol. 757, no. 1-2, pp. 102-183, 2005.

[6] K. Adcox, S. S. Adler, S. Afanasiev et al., "Formation of dense partonic matter in relativistic nucleus-nucleus collisions at RHIC: experimental evaluation by the PHENIX Collaboration," Nuclear Physics A, vol. 757, no. 1-2, pp. 184-283, 2005.

[7] F. Karsch and E. Laermann, "Examples of soft physics observables in the ALICE experiment," in Quark-Gluon Plasma, R. C. Hwa and X. N. Wang, Eds., World Scientific, Singapore, 2004.

[8] Quark Matter 2001: Proceedings of the 15th International Conference on Ultra Relativistic Nucleus-Nucleus Collisions, vol. 698 of Nuclear Physics A, North-Holland, Amsterdam, The Netherlands, 2002.

[9] E. V. Shuryak, "Quantum chromodynamics and the theory of superdense matter," Physics Reports, vol. 61, no. 2, pp. 71-158, 1980.

[10] K. Kajantie and H. I. Miettinen, "Temperature measurement of quark-gluon plasma formed in high energy nucleus-nucleus collisions," Zeitschrift für Physik C, vol. 9, no. 4, pp. 341-345, 1981.

[11] G. Domokos and J. Goldman, "Quark-matter diagonstics," Physical Review D, vol. 23, p. 203, 1981.

[12] L. D. McLerran and T. Toimela, "Photon and dilepton emission from the quark-gluon plasma: some general considerations," Physical Review D, vol. 31, no. 3, pp. 545-563, 1985.

[13] C. Gale and J. I. Kapusta, "Vector dominance model at finite temperature," Nuclear Physics B, vol. 357, no. 1, pp. 65-89, 1991.

[14] J. Alam, S. Raha, and B. Sinha, "Electromagnetic probes of quark gluon plasma," Physics Reports, vol. 273, no. 5-6, pp. 243-362, 1996.

[15] T. Peitzmann and M. H. Thoma, "Direct photons from relativistic heavy-ion collisions," Physics Reports, vol. 364, no. 3, pp. 175246, 2002.

[16] W. Cassing and E. L. Bratkovskaya, "Hadronic and electromagnetic probes of hot and dense nuclear matter," Physics Report, vol. 308, no. 2, pp. 65-233, 1999.

[17] P. Aurenche, "Photon production inheavy ion collisions," http://arxiv.org/abs/hep-ph/0201011.

[18] J. Kapusta, P. Lichard, and D. Seibert, "High-energy photons from quark-gluon plasma versus hot hadronic gas," Physical Review D, vol. 44, no. 9, pp. 2774-2788, 1991.

[19] J. Kapusta, P. Lichard, and D. Seibert, "Erratum: high-energy photons from quark-gluon plasma versus hot hadronic gas," Physical Review D, vol. 47, no. 9, p. 4171, 1993.

[20] R. Baier, H. Nakkagawa, A. Niégawa, and K. Redlich, "Production rate of hard thermal photons and screening of quark mass singularity," Zeitschrift für Physik C, vol. 53, no. 3, pp. 433-438, 1992.
[21] J. Alam, S. Sarkar, T. Hatsuda, T. K. Nayak, and B. Sinha, "Photons from $\mathrm{Pb}-\mathrm{Pb}$ collisions at ultrarelativistic energies," Physical Review C, vol. 63, no. 2, Article ID 021901, 5 pages, 2001.

[22] M. M. Aggarwal, A. Agnihotri, Z. Ahammed et al., "Observation of direct photons in central $158 \mathrm{~A} \mathrm{GeV}{ }^{208} \mathrm{P}^{208} \mathrm{~b}+\mathrm{Pb}$ collisions," Physical Review Letters, vol. 85, no. 17, pp. 3595-3599, 2000.

[23] M. M. Aggarwal, A. Agnihotri, Z. Ahammed et al., "Direct photon production in $158 \mathrm{~A} \mathrm{GeV}{ }^{208} \mathrm{~Pb}+{ }^{208} \mathrm{~Pb}$ collisions," http: //arxiv.org/abs/nucl-ex/0006007.

[24] M. M. Aggarwal, A. Agnihotri, Z. Ahammed et al., "Scaling of particle and transverse energy production in ${ }^{208} \mathrm{~Pb}+{ }^{208} \mathrm{~Pb}$ collisions at 158 A GeV," European Physical Journal C, vol. 18, pp. 651-663, 2001.

[25] M. M. Aggarwal, Z. Ahammed, A. L. S. Angelis et al., "Interferometry of direct photons in central ${ }^{208} \mathrm{~Pb}+{ }^{208} \mathrm{~Pb}$ collisions at 158A GeV," Physical Review Letters, vol. 93, no. 2, Article ID 022301, 5 pages, 2004.

[26] R. Albrecht, V. Antonenko, T. C. Awes et al., "Limits on the production of direct photons in $200 \mathrm{~A} \mathrm{GeV}^{32} \mathrm{~S}+\mathrm{Au}$ collisions," Physical Review Letters, vol. 76, no. 19, pp. 3506-3509, 1996.

[27] D. P. Morrison, Y. Akiba, O. Alford et al., "The PHENIX experiment at RHIC," Nuclear Physics A, vol. 638, no. 1-2, pp. $565 c-569 c, 1998$.

[28] S. Y. Wang and D. Boyanovsky, "Enhanced photon production from quark-gluon plasma: finite-lifetime effect," Physical Review D, vol. 63, no. 5, Article ID 051702, 5 pages, 2001.

[29] D. Boyanovsky and H. J. de Vega, "Are direct photons a clean signal of a thermalized quark-gluon plasma?" Physical Review $D$, vol. 68, no. 6, Article ID 065018, 26 pages, 2003.

[30] S. Sarkar, P. Roy, J. Alam, and B. Sinha, "Photons from $\mathrm{Pb}+\mathrm{Pb}$ and $\mathrm{S}+\mathrm{Au}$ collisions at ultrarelativistic energies," Physical Review C, vol. 60, no. 5, Article ID 054907, 6 pages, 1999.

[31] D. K. Srivastava and B. Sinha, "Single photons from S + $\mathrm{Au}$ collisions at CERN super proton synchrotron and quarkhadron phase transition," Physical Review Letters, vol. 73, no. 18, pp. 2421-2424, 1994.

[32] R. D. Pisarski, "Computing finite-temperature loops with ease," Nuclear Physics B, vol. 309, no. 3, pp. 476-492, 1988.

[33] R. D. Pisarski, "Scattering amplitudes in hot gauge theories," Physical Review Letters, vol. 63, pp. 1129-1132, 1989.

[34] E. Braaten and R. D. Pisarski, "Soft amplitudes in hot gauge theories: a general analysis," Nuclear Physics B, vol. 337, no. 3, pp. 569-634, 1990.

[35] Y. Kumar and S. S. Singh, "Dilepton production in finite baryonic quark-gluon plasma," Canadian Journal of Physics, vol. 90, no. 10, pp. 955-961, 2012.

[36] R. Ramanathan, Y. K. Mathur, K. K. Gupta, and A. K. Jha, "Simple statistical model for analysis of quark-gluon plasma droplet (fireball) formation," Physical Review C, vol. 70, no. 2, Article ID 061408, 4 pages, 2004.

[37] R. Ramanathan, K. K. Gupta, A. K. Jha, and S. S. Singh, “The interfacial surface tension of a quark-gluon plasma fireball in a hadronic medium," Pramana, vol. 68, no. 5, pp. 757-768, 2007.

[38] R. Balian and C. Block, "Distribution of eigenfrequencies for the wave equation in a finite domain: I. Three-dimensional problem with smooth boundary surface," Annals of Physics, vol. 64, no. 2, pp. 401-447, 1970.

[39] I. Mardor and B. Svetitsky, "Bubble free energy at the quarkhadron phase transition," Physical Review D, vol. 44, no. 3, pp. 878-886, 1991. 
[40] G. Neerguard and J. Madsen, "Free energy of bubbles and droplets in the quark-hadron phase transition," Physical Review $D$, vol. 60, no. 5, Article ID 054011, 12 pages, 1999.

[41] M. G. Mustafa, D. K. Srivastava, and B. Sinha, "Effect of colour singletness of quark-gluon plasma in quark-hadron phase transition," The European Physical Journal C, vol. 5, no. 4, pp. 711-718, 1998.

[42] A. Peshier, B. Kämpfer, O. P. Pavlenko, and G. Soff, "An effective model of the quark-gluon plasma with thermal parton masses," Physics Letters B, vol. 337, no. 3-4, pp. 235-239, 1994.

[43] H. A. Bethe, "Nuclear physics: B nuclear dynamics, theoretical," Reviews of Modern Physics, vol. 9, no. 2, pp. 69-244, 1937.

[44] E. Fermi, "Eine statistische Methode zur Bestimmung einiger Eigenschaften des Atoms und ihre Anwendung auf die Theorie des periodischen Systems der Elemente," Zeitschrift für Physik, vol. 48 , no. 1-2, pp. 73-79, 1928.

[45] L. H. Thomas, "The calculation of atomic fields," Proceedings of the Cambridge Philosophical Society, vol. 23, no. 5, pp. 542-548, 1927.

[46] H. Weyl, Über die Asymptotische Verteilung der Eigenwerte, Nachrichten der Akademie der Wissenschaften, Göttingen, Germany, 1911.

[47] G. D. Moore, "Electromagnetic emission and energy loss in the QGP, Journal of Physics G, vol. 30, no. 8, 2004.

[48] V. Ruuskanen, in Quark-Gluon Plasma, R. C. Hwa, Ed., World Scientific, Singapore, 1991.

[49] C. T. Traxler, H. Vija, and M. H. Thoma, "Hard photon production rate of a quark-gluon plasma at finite quark chemical potential," Physics Letters B, vol. 346, no. 3-4, pp. 329-334, 1995.

[50] P. Aurenche, F. Elis, R. Kobes, and H. Zaraket, "Bremsstrahlung and photon production in thermal QCD," Physical Review D, vol. 58, Article ID 085003, 17 pages, 1998.

[51] D. K. Srivastava, "Single photons, dileptons and hadrons from relativistic heavy ion collisions and quark-hadron phase transition," Pramana, vol. 57, no. 2-3, pp. 235-249, 2001.

[52] D. K. Srivastava, "Photon production in relativistic heavy-ion collisions using rates with two-loop calculations from quark matter," The European Physical Journal C, vol. 10, pp. 487-490, 1999.

[53] F. D. Steffen and M. H. Thoma, "Hard thermal photon production in relativistic heavy ion collisions," Physics Letters B, vol. 510, pp. 98-106, 2001.

[54] T. Renk, "Emission of thermal photons and the equilibration time in heavy-ion collisions," Physical Review C, vol. 67, no. 6, Article ID 064901, 8 pages, 2003.

[55] C. T. Traxler and M. H. Thoma, "Photon emission from a parton gas at chemical nonequilibrium," Physical Review C, vol. 53, no. 3, pp. 1348-1352, 1996.

[56] J. Y. Ollitrault, "Relativistic hydrodynamics for heavy-ion collisions," European Journal of Physics, vol. 29, no. 2, p. 275, 2008.

[57] M. Martinez and M. Strickland, "Pre-equilibrium dilepton production from an anisotropic quark-gluon plasma," Physical Review C, vol. 78, no. 3, Article ID 034917, 19 pages, 2008.

[58] A. Adare, S. Afanasiev, C. Aidala et al., "Enhanced production of direct photons in $\mathrm{Au}+\mathrm{Au}$ collisions at $\sqrt{S_{N N}}=200 \mathrm{GeV}$ and implications for the initial temperature," Physical Review Letters, vol. 104, no. 13, Article ID 132301, 2010.

[59] H. B. Büsching, "Highlights from the PHENIX experimentpart 2," Nuclear Physics A, vol. 774, pp. 103-112, 2006.
[60] T. Isobe, H. Gustafsson, E. Haslum et al., "Systematic study of high-pT direct photon production with the PHENIX experiment at RHIC," Journal of Physics G, vol. 34, no. 8, 2007.

[61] J. Alam, J. K. Nayak, P. Roy, A. K. Dutt-Mazumder, and B. Sinha, "Thermal radiation from $\mathrm{Au}+\mathrm{Au}$ collisions at $\sqrt{S_{N N}}=200 \mathrm{GeV}$ energy," Journal of Physics G, vol. 34, no. 5, p. 871, 2007.

[62] C. Gale, S. Turbide, E. Frodermann, and U. Heinz, "Photons from nuclear collisions at RHIC energies," Journal of Physics G, vol. 35, no. 10, Article ID 104119, 2008.

[63] K. Dusling and I. Zahed, "Thermal photons from heavy ion collisions: a spectral function approach," Physical Review C, vol. 82, no. 5, Article ID 054909, 11 pages, 2010. 

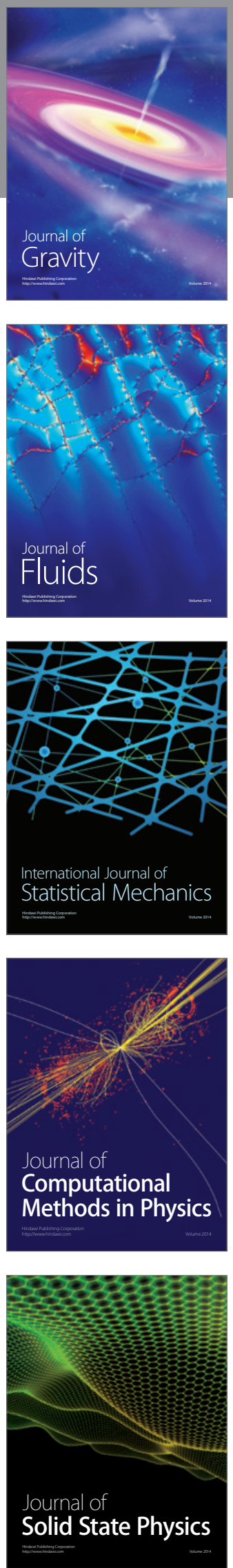

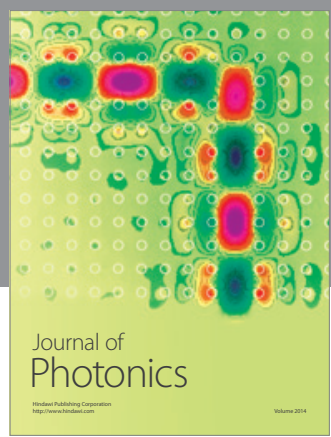

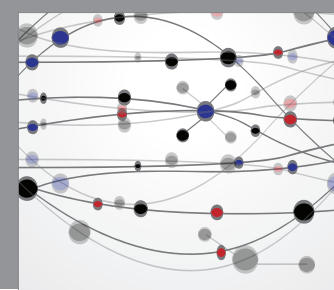

The Scientific World Journal

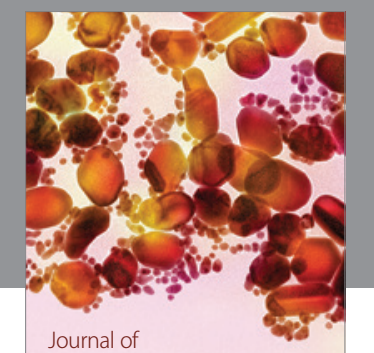

Soft Matter
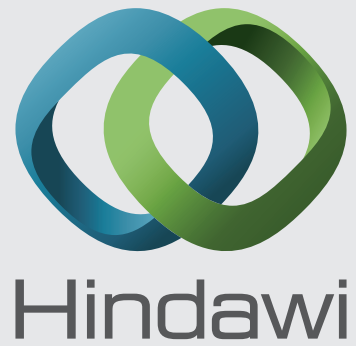

Submit your manuscripts at

http://www.hindawi.com
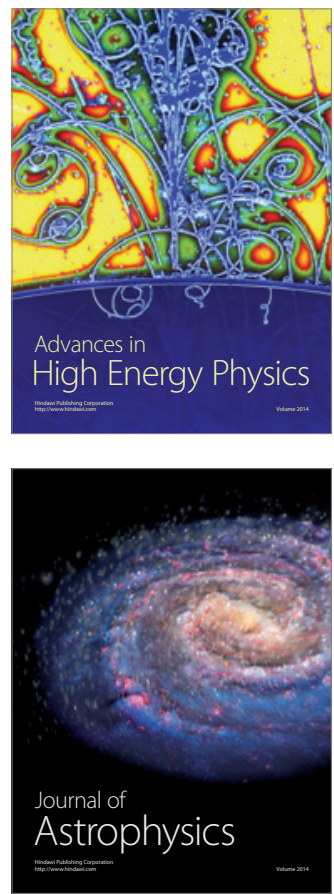
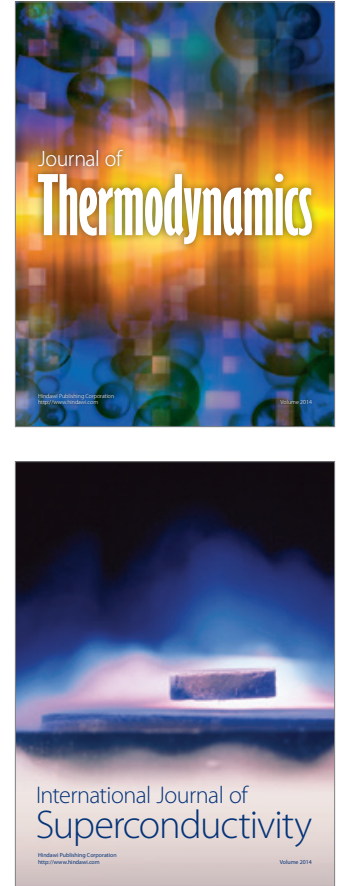
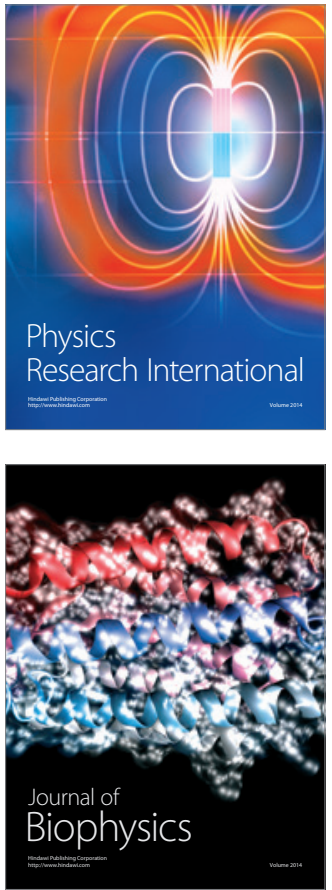
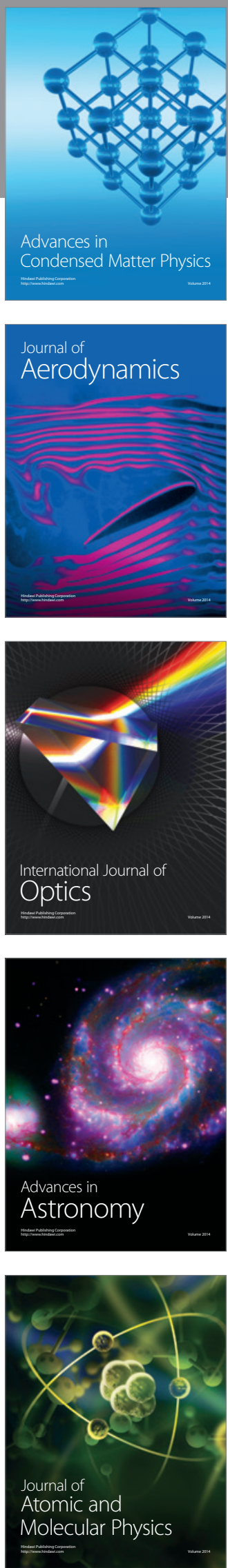